Human \& Animal Health

Vol. 59: e16150477, January-December 2016 http://dx.doi.org/10.1590/1678-4324-2016150477

ISSN 1678-4324 Online Edition
BRAZILIAN ARCHIVES OF BIOLOGY AND TECHNOLOGY

A N INTERNATIONAL JOURNAL

\title{
Liposome and Their Applications in Cancer Therapy
}

\author{
Himanshu Pandey ${ }^{2}$, Radha Rani $^{1}$, Vishnu Agarwal $^{1} *$ \\ ${ }^{1}$ Department of Biotechnology, Motilal Nehru National Institute of Technology (MNNIT), Allahabad, India ${ }^{2}$ \\ Faculty of Pharmaceutical Sciences, Sam Higginbottom Institute of Agriculture Technology \& Sciences, Allahabad, \\ India.
}

\begin{abstract}
Liposomes, the vesicles of phospholipid bilayer, can encapsulate both hydrophilic and lipophilic drugs and protect them from degradation. Liposomes have been extensively studied and continue to create intense interest in research since their discovery in the mid-1960s. Since then, liposomes have been considered to be the most successful nanocarriers for drug deliver and have made their way to the market. Currently, a number of liposomal formulations are on the marker for cancer treatment and many more are in pipe line. This review discusses about the liposome components, methods of preparation, drug encapsulation mechanism and the potential therapeutic applications of liposomes in cancer therapy.
\end{abstract}

Keywords: Liposomes, Drug delivery, Cancer, Doxil, LipoDox, Myocet

*Author for correspondence: vishnua@mnnit.ac.in 
Agarwal, $\mathrm{V}$ et al.

\section{INTRODUCTION}

Cancer is the major health concern of the century because of the leading cause of death worldwide (Fitzmaurice et al. 2015; Torre et al. 2015). It kills millions of people very year and its burden continues to rise at an alarming rate globally (Stewart and Wild 2014). Cancer is the uncontrolled growth of cells, which occurs due to the accumulation of genetic mutations and aberrant signaling of various pathways related to the growth and survival of the cells (Bhardwaj et al. 2014; Tyagi et al. 2014; Deshmukh et al. 2015; Srivastava et al. 2015a; Srivastava et al. 2015b). The complexity at genetic and phenotypic levels in cancer cells leads to the clinical diversity and therapeutic resistance in cancer cells. Chemotherapy is most commonly used treatment among a variety of approaches currently being used for the treatment of cancer, which, however, kpossesses several limitations and side effects (MacDonald 2009; Ramirez et al. 2009; Iwamoto 2013). According to an estimate, more than $90 \%$ cancer drugs exhibit poor bioavailability and pharmacokinetics (Iwamoto 2013). Therefore, there is a prerequisite to develop appropriate drug delivery systems, which can improve the bioavailability, pharmacokinetic properties and can deliver the active drug molecules to the site of action, without affecting the healthy cells.

To overcome the limitations of conventional chemotherapy, a number of nanocarrier delivery systems have been developed and extensively used for drug delivery to cancer cells (Tyagi et al. 2011; Tyagi et al. 2013; Arora et al. 2015).

Nanocarriers have larger surface area as compared to bigger particles, which can be easily modified to encapsulate large amount of drug, to increase the blood circulation time and to enhance the accumulation of drugs in solid tumors via the enhanced permeability and retention (EPR) effect as well as selective targeting of tumor cells (Tyagi and Ghosh 2011; Allen and Cullis 2013; Bozzuto and Molinari 2015).

Nanocarriers also improve the solubility, bioavailability and pharmacokinetics properties of chemotherapeutics (Gregoriadis and Florence 1993; Bozzuto and Molinari 2015; Pattni et al. 2015). Currently, a variety of nanocarriers such as liposomes, polymeric nanoparticles, micelles, nanotubes, etc are already in the market, or under research and evaluation for cancer treatment (Sutradhar and Lutful 2014). This review summarizes the types of methods used for the preparation of liposomes, mechanism of drug loading and potential therapeutic applications in cancer therapy and provides current information on the liposomal products, which are either in clinical use, or clinical trials.

\section{LIPOSOMES}

Bangham (Bangham et al. 1965) for the first time observed that phospholipids in aqueous medium forms closed bilayer structures. Later, these closed bilayer structures were termed as liposomes by Sessa (Sessa and Weissmann 1968). The liposome comprises of an aqueous compartment surrounded by one, or more lipid bilayers (Gregoriadis and Florence 1993; Pattni et al. 2015).

Initially, liposomes were used to study the physical behavior of biological membranes like lipids orientation in bilayer, physiochemical characterization of lipids and ion transport across bio membranes (Bangham 1972; Gregoriadis and Florence 1993). However, now liposomes are extensively used for drug delivery as they meet all the requirements of a good delivery vehicle. Liposomes are biodegradable, biocompatible, and stable in colloidal solutions (Akbarzadeh et al. 2013; Allen and Cullis 2013). Liposomes protect the drug from degradation and reduce drug-related nonspecific toxicity and can be produced and formulated easily for the target specific delivery (Bitounis et al. 2012; Bozzuto and Molinari 2015).

\section{TYPES OF LIPOSOMES}

Liposomes can be classified on the basis of size and the number of phospholipid membrane layers (Akbarzadeh et al. 2013; Pattni et al. 2015) as depicted in Figure 1.

Multilamellar Vesicles (MLV): These liposomes are composed of a number of concentric phospholipid bilayer membrane separated by aqueous phase. These are big in size and may be up to $5 \mu \mathrm{m}$. 
Small Unilamellar Vesicles (SUV): These liposomes are composed of aqueous compartment enclosed by a single lipid bilayer. The size of these liposomes may be in the range of 20-100 nm.
Large Unilamellar Vesicle (LUV): These liposomes are also composed of a single lipid bilayer surrounding aqueous compartment. The size of these liposomes is in the range of 100$250 \mathrm{~nm}$.

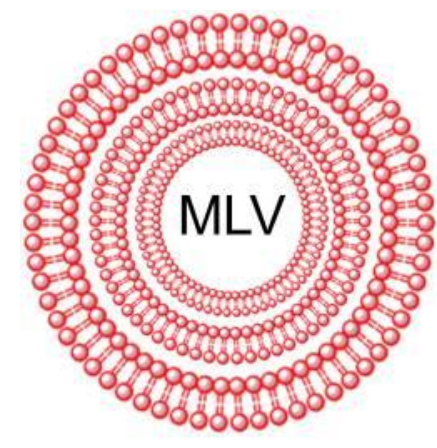

1-5 $\mu \mathrm{M}$

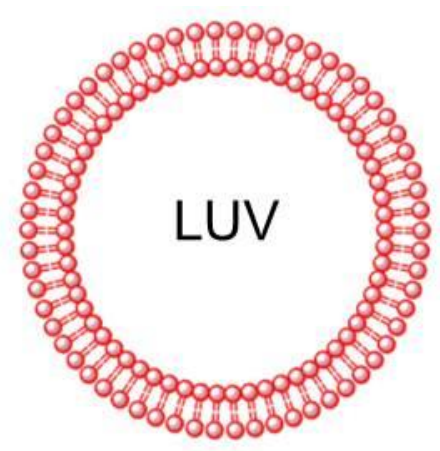

$100-250 \mathrm{~nm}$

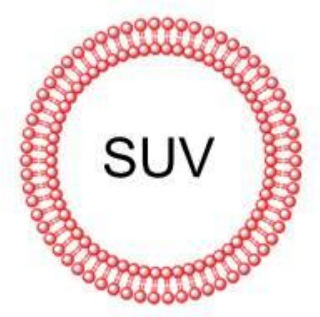

20-100 nm

Figure 1- Classification of liposomes based on the lamellarity: (A) Multilamellar Vesicles (MLV) is composed of many lipid bilayers and ranges from 1-5 $\mu \mathrm{m}$ in size. (B) Large Unilamellar Vesicle (LUV) is in the size range of 100-250 nm with single lipid bilayer. (C) Small Unilamellar Vesicles (SUV) consists of a single phospholipid bilayer surrounding the aqueous phase with size range $20-100 \mathrm{~nm}$.

\section{COMPONENTS OF LIPOSOMES}

The major components of liposomes are phospholipids and cholesterol, major constituents of natural bio membranes. The chemical properties of these lipids control the behavior of liposomes.

\section{Phospholipids}

The most common phospholipids used for the preparation of liposomes are natural (egg, or soy) phosphatidylcholine, or synthetic phosphatidylcholine (PC). The natural phospholipids such as egg, or soyabean phospholipids contain substantial levels of polyunsaturated fatty acids making them less stable than the synthetic equivalents (Jing Li et al. 2015). The molar percentage of phospholipids varies from 55 to $100 \%$ of total liposomal components (Bozzuto and Molinari 2015; Jing Li et al. 2015). The most common phospholipid component of liposomes is 2distearoyl-sn-glycerophosphocholine (DSPC). The chemical structure of DSPC is presented in Figure 2A. This molecule is composed of a polar phosphate head group and the hydrophobic portion composed of hydrocarbon chains. The hydrocarbon chains form the interior and the polar head forms the exterior of liposomes bilayer. The head portion can be modified by attaching a functional group. The 1,2-distearoylsn-glycero-3-phosphoethanolamine (DSPE) is an example of a functional phospholipid used to conjugate other polymers like polyethylene glycol (PEG) (Laouini et al. 2012; MarquesGallego and de Kroon 2014; Jing Li et al. 2015) (Figure 2B). The type, molar percentage and packing orientation of phospholipids determine the ultimate shape and size of the liposomes (Farge and Devaux 1992; Jing Li et al. 2015). The orientation of phospholipids in liposome bilayer depends upon the length of lipid molecules and the size of head groups (Laouini et al. 2012; Jing Li et al. 2015).

The phase transition temperature (Tc) of phospholipids is also an important criterion to choose phospholipid for the preparation of liposomes (Laouini et al. 2012; Bozzuto and Molinari 2015; Jing Li et al. 2015). The phase transition temperature is defined as the temperature at which the lipid physical state converts from an ordered gel phase to a disordered liquid crystalline phase. The conversion of phases depends on hydrocarbon chain length, degree of saturation, charge, and head group species (Bitounis et al. 2012; Laouini et al. 2012; Bozzuto and Molinari 2015). 
The use of phospholipids with higher phase transition temperatures generates bilayers, which are more stable (Ellens et al. 1986). This decreases the possibility for premature leakage of encapsulated components; however, considerations must be made to ensure that encapsulated drugs can still escape the liposomes once they reach the target site of action. On the other hand, if the phase transition temperature of the selected phospholipids is too high, denaturation of the encapsulated drugs may occur during the sizing, or loading processes (Bitounis et al. 2012; Laouini et al. 2012; Jing Li et al. 2015). Therefore, a good balance must be met to guarantee that the selected lipids have phase transition temperatures that prevent premature leakage of components but enable processing to occur at temperatures that are harmless to all liposomal components.

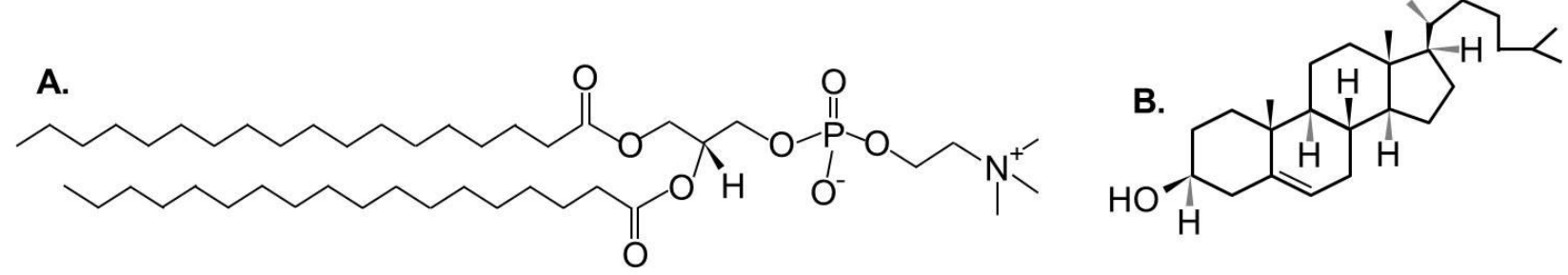

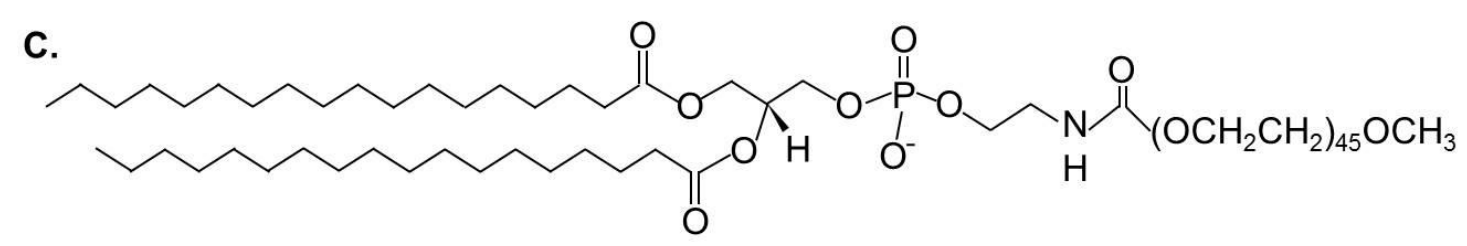

Figure 2- Chemical structures of common liposomal components: (A) 1, 2-distearoyl-sn-glycerophosphocholine (DSPC) (B) Cholesterol and (C) 1, 2-distearoyl-sn-glycero-3-phosphoethanolamine polyethylene glycol (DSPEPEG).

\section{Cholesterol}

The rotational freedom because of flip-flop movements in phospholipids generates liposomes of leaky properties. Cholesterol is the main component added in the liposomal formulations to stabilize the bilayer of liposomes (Laouini et al. 2012; Magarkar et al. 2014). Depending upon the rigidity and fluidity of bilayer, the molar percentage of cholesterol varies from $30-45 \%$ of total liposomes components ( Kirby and Gregoriadis 1980; Laouini et al. 2012) as it provides membrane fluidity, elasticity, permeability and stability to liposomes (Kirby and Gregoriadis 1980; Magarkar et al. 2014). The chemical structure of cholesterol is presented in Figure 2C.

The polar head of cholesterol is aligned with the polar head of the phospholipids of lipid bilayer. Due to hydrophobic properties of cholesterol, it resides in the interior portion of lipid bilayers and serves to fill the gap created because of imperfect packing of phospholipid molecules. The packing of cholesterol within phospholipid bilayers prevents the flip-flop of membrane components and the movement across the membranes (Kirby and Gregoriadis 1980; Farge and Devaux 1992).

Cholesterol also provides the rigidity to liposomes as it prevents the phase transition of lipid bilayers, and thus reduces the leakage of encapsulated drugs (Manes and Martinez 2004). Therefore, the percentage of cholesterol used for the preparation of liposomes also affects the ultimate phase transition temperature of the bilayer. Some studies have suggested that the cholesterol also helps in protecting the lipid bilayer from hydrolytic degradation (Simon et al. 1982). Depending on the final application of liposomes, many other components in addition to phospholipid and cholesterol have been used. Depending upon the component used, liposomes can be neutral, negative, or positively charged. The charge on the surface of liposomes plays an important role in deciding the fate and application of the liposomes (Miller et al. 1998; Tyagi et al. 2011). PEG is the other commonly used liposome component typically incorporated to increase the blood circulation times because of its stealth properties and has shown broad applications (Miller et al. 1998; Immordino et al. 2006; Tyagi and Ghosh 2011; Tyagi et al. 2013). 


\section{METHODS OF PREPARATION}

There are several methods for the preparation of liposomes such as solvent removal, detergent removal, emulsion removal and ethanol injection (Laouini et al. 2012; Bozzuto and Molinari 2015). The type of preparation methods influences the properties of liposomes, including their shape, size, stability and drug loading efficiency. Thin lipid film hydration, or solvent removal method is the most common and first described method for liposome preparations (Akbarzadeh et al. 2013; Bozzuto and Molinari 2015). Briefly, the lipids are dissolved in chloroform and/or methanol mixture. The concentration of lipids is typically in the range of $10-20 \mathrm{mgmL}^{-1}$ depending on the solubility of lipids.

The solvent is subsequently removed by a rotary evaporator under reduced pressure to produce a thin film of lipids. The thin film so formed is desiccated for required time, followed by hydration. Hydration of the dry lipid film is accomplished by adding aqueous solution, which has the osmolarity in physiological range. After completion of hydration, the liposomes of multilamellar vesicles (LMV) in the size range of $200-1000 \mathrm{~nm}$ are produced (Laouini et al. 2012; Akbarzadeh et al. 2013). These MLVs are broken down into smaller liposomes by sonication, or extrusion.

Sonication is generally performed in water bath type sonicators and the temperature of water is maintained above the Tc of lipids. Sonic waves disrupt the outer layers of gaint liposomes and produce small unilamellar vesicles (SUV), ranging between 20-100 $\mathrm{nm}$ in diameter (Laouini et al. 2012; Akbarzadeh et al. 2013).

The final size of liposomes not only depends upon the sonication time and energy but also upon many factors, including lipid composition, concentration and suspension volume. Alternatively, the liposomes are passed through the extrusion assembly containing a polycarbonate membrane of definite size to reduce the size of MLVs. This process is also performed under high pressure and at a temperature above the lipid Tc. The final size of extruded liposomes tends to be close to the filter pore size. Extrusion through filters with $100 \mathrm{~nm}$ pores yields large unilamellar vesicles (LUV) of reproducible size.

\section{ENCAPSULATION OF DRUGS INTO LIPOSOMES}

The methods of drug encapsulation in to the liposomes can be divided into two sub groups. The passive loading in which drug encapsulation occur during the vesicle formation process and the active loading in which drug is entrapped after the formation of vesicles.

\section{Passive loading}

Passive loading is to encapsulate the drug during the formation of liposomes. The hydrophilic drugs are loaded within the internal core of the liposomes by mixing with the hydrating buffer used to hydrate the thin lipid film during the formation of liposomes. Lipophilic drugs are mixed with other liposome components during the preparation of thin dry film of lipids and ultimately loaded into lipid bilayers. The unentrapped drug molecules are removed from liposome suspension by dialysis, or gel-filtration chromatography (Tyagi et al. 2011; Tyagi et al. 2013).

The encapsulation efficiency depends on lipid concentration, liposome size, choice of lipids, etc. The encapsulation efficiency of watersoluble compounds, which do not interact with the lipid bilayer, is relatively low if loaded by passive method and proportional to the aqueous volume enclosed in the liposomes (Tyagi et al. 2013). Large vesicles will have higher encapsulation efficiency than small vesicles (Akbarzadeh et al. 2013). While the drug that interacts with lipid bilayer, such as lipophilic compound, normally have better encapsulation rate.

Therefore, several strategies have been developed to improve the encapsulation efficiency by linking lipophilic chain to drug molecule to increase its lipophilicity and better partition into the lipid bilayer (Sutradhar and Lutful 2014; Bozzuto and Molinari 2015). Choice of lipid composition is also critical for better loading efficiently by this method. For example, to load highly negatively charged nucleotide compounds, such as antisense or siRNA, selection of cationic lipid will greatly improve the encapsulation efficiency due to enhanced drug/lipid interaction. 


\section{Active loading}

Certain weakly acidic, or alkaline drug molecules are loaded into preformed liposomes by active loading, or remote loading method. This process is driven by an electrochemical potential created by the $\mathrm{pH}$, or ion gradients established across the lipid bilayer of the liposomes (Akbarzadeh et al. 2013; Bozzuto and Molinari 2015). The $\mathrm{pH}$, or ion gradients are created during the liposomes preparation by using a buffer of specified $\mathrm{pH}$ and ion concentration. The external $\mathrm{pH}$ of liposomes is then exchanged with another buffer of different $\mathrm{pH}$, or ion concentration through dialysis, or size exclusion chromatography. After creating the $\mathrm{pH}$ gradient across the liposomes membranes, drug is loaded by mixing with liposomes typically at a temperature above the phase transition temperature of the lipids to ensure the fluidity and efficient transport across the bilayer. The drug molecules interact with the ions within liposomes and get charged. The charged drug molecules are not capable to come out and remain entrapped within liposome core.
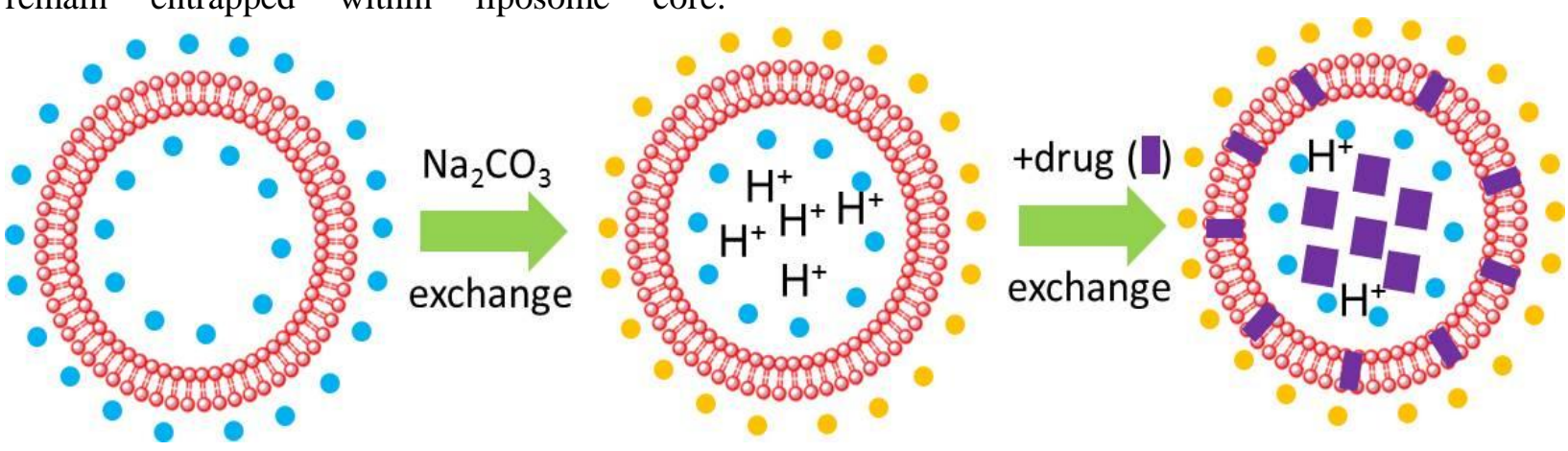

Doxil $^{\mathrm{TM}}$, liposomal doxorubicin, is the ideal example of the active loading by $\mathrm{pH}$ gradient method (Lasic et al. 1992; Haran et al. 1993). The active loading of doxorubicin by $\mathrm{pH}$ gradient method is shown in Figure 3. As shown in the figure, when the gradient of citrate buffer liposome is more than 1000 times to the citrate buffer of medium then a $\mathrm{pH}$ gradient is created. Doxorubicin, a weak base, is in equilibrium between an ionized state and a non-ionized state. The latter can cross the lipid bilayer, become ionized in the high proton intraliposome environment and leads to high efficient accumulation of doxorubicin inside the liposome. The another example of $\mathrm{pH}$-gradient method is the loading of chloroquine diphosphate into liposomes (Qiu et al. 2008). In another study, oxymatrine- major active alkaloid constituent extracted from the traditional Chinese herb medicine Sophora flavescens, used for treating hepatitis B in clinical therapy in China, was also loaded into liposomes by $\mathrm{pH}$ gradient method (Du and Deng 2006).

Figure 3- Active loading of drugs into liposomes: Liposomes were prepared by hydrating in citrate buffer $(\bigcirc)$ and then external phase was exchanged with $\mathrm{Na} 2 \mathrm{CO} 3(\mathrm{O})$ to create a $\mathrm{pH}$ gradient. (C) The neutral form of the externally

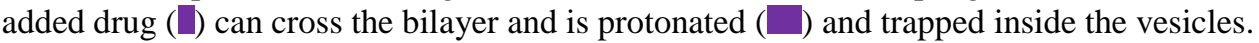

\section{APPLICATIONS OF LIPOSOMES IN CANCER}

Liposomes have been successfully used in cancer therapy. Although, the application of liposomes in the field of cancer therapeutics has been extensively studied and deserves a broad assessment but this is outside the scope of this review. However, the most successful applications of liposomes in cancer therapeutics are discussed here. A number of different liposomal formulations of anti-cancer agents have been shown to deliver the drug at the site 
drug doxorubicin commercialized by Johnson \& Johnson. In 2011, an imbalance between the demand and supply of Doxil was observed as the manufacturing unit was shut down temporarily due to some quality control issues (Berger et al. 2014; Chou et al. 2015). To address the Doxil shortage in USA, FDA allowed temporary importation of LipoDox. LipoDox is the same liposomal formulation as Doxil in USA and made in India by Sun Pharma and in 2013, FDA approved the first generic version of Doxil, made by Sun Pharma (Berger et al. 2014; Chou et al. 2015).

In a study, it was observed that Doxil was also active against refractory ovarian cancer, and later approved by the FDA for the treatment of recurrent ovarian cancer also (Muggia 1997; Barenholz 2012). Recently, it has been approved for the treatment of breast cancer (Barenholz 2012) in USA and for the treatment of multiple myeloma in combination with velcade in Europe and Canada (Blade et al. 2011; Barenholz 2012). DaunoXome, the registered trademark of Galen, is the liposomal formulation of daunorubicin approved by the FDA for the treatment of AIDS related kaposi's sarcoma (Cooley et al. 2007; Petre and Dittmer 2007). Myocet, the registered trade mark of Cephalon, is a non-PEGylated liposomal formulation of doxorubicin. Myocet in combination with cyclophosphamide was approved for the treatment of metastatic breast cancer in Europe but was not yet approved by the FDA for use in the United States (Batist et al. 2001).

The Sopherion Therapeutics in the United States and Canada is conducting a pivotal phase III global registrational trial of Myocet in combination with Herceptin (trastuzumab) and Taxol (paclitaxel) for the treatment of highly aggressive HER2-positive metastatic breast cancer (Baselga et al. 2014). The liposomal formulation of vincristine made by Talon was registered under trade name of Marqibo. Marqibo was approved in 2012 by the FDA for the treatment of acute lymphoblastic leukemia (Sarris et al. 2000; Rodriguez et al. 2009).

Celator Pharmaceuticals Inc developed CPX351, a liposomal formulation of cytarabine and daunorubicin. The CPX-351 showed promising results in phase III clinical trial on the patients with secondary acute myeloid leukemia (AML) by improving the induction response over $40 \%$ (Riviere et al. 2011; Cortes et al. 2015).
Previously in phase II trial, CPX-351 had already showed a survival benefits and the data on over survival could be expected in the first quarter of 2016 (Lancet et al. 2014). Another liposomal formulation of Celator contains irinotecan $\mathrm{Hcl}$ and floxuridine and registered as CPX-1. The CPX-1 completed phase II clinical trial on the patients with advanced colorectal cancer (Batist et al. 2009). MM-398 is a liposomal sphere encapsulating irinotecan developed by Merrimack pharma. MM-398 is being evaluated in the clinical trials for its ability to treat various cancers, which are resistant to chemotherapy such as pancreatic, colorectal, lung and glioma (Ko et al. 2013; Roy et al. 2013; Saif 2014). Another liposomal formulation developed by Merrimack pharma is MM-302, which encapsulates doxorubicin. MM302 is designed for selective uptake of drug into tumor cells while sparing off healthy tissues. MM-302 contains a novel antibody-drug conjugated on the surface that specifically targets cancer cells overexpressing the HER2 receptor. Currently, MM-302 is being evaluated in phase I clinical trials for its ability to treat advanced metastatic HER2-positive breast cancer (Geretti et al. 2015). MBP-426 is transferrin receptor targeted liposomal formulation of oxaliplatin designed by Mebiopharm. MBP-426 is being evaluated in phase II clinical trial for the treatment of patients with gastric cancer (Suzuki et al. 2008; Goldberg et al. 2013).

Lipoplatin is the liposomal formulation of cisplatin designed by Regulon Inc. and currently, it is being evaluated in phase III clinical trial for the patients with non-small cell lung cancer (Fantini et al. 2011). Another liposomal formulation Stimuvax is designed as anti-MUC1 cancer vaccine by Oncothyreon to treat non-small cell lung cancer and presently is in phase III clinical trial (Bradbury and Shepherd 2008; Fantini et al. 2011; Broglio et al. 2014). The thermo sensitive liposomal formulation of doxorubicin, called ThermoDox (Celsion) is under phase III clinical trial to treat the patients with primary hepatocellular carcinoma, in phase II for refractory chest wall breast cancer and colorectal liver metastasis (Poon and Borys 2011; Staruch et al. 2011). 


\section{CONCLUSION}

Liposomes have revolutionized cancer therapy by their broad clinical applications. Liposomes overcome the limitations of conventional chemotherapy by improving the bioavailability and stability of the drug molecules and minimizing side effects by sitespecific targeted delivery of the drugs. Liposomes were the first nanotechnology-based drug delivery systems approved for the clinical applications because of their biocompatibility and biodegradability like features. Some liposome-based drug delivery systems are already in the market and many more are undergoing research and clinical trials. So far, liposomes have established themselves in nanocarriers-based drug delivery systems as evident by the successful clinical applications of liposomal formulations in anti-cancer therapy.

\section{REFERENCES}

Akbarzadeh A, Rezaei-Sadabady R, Davaran S, Joo SW, Zarghami N, Hanifehpour Y, et al. Liposome: classification, preparation, and applications. Nanoscale Res Lett. 2013; 8: 102108.

Allen TM and Cullis PR. Liposomal drug delivery systems: from concept to clinical applications. Adv Drug Deliv Rev. 2013; 65: 36-48.

Arora S, Tyagi N, Bhardwaj A, Rusu L, Palanki R, Vig K, et al. Silver nanoparticles protect human keratinocytes against UVB radiation-induced DNA damage and apoptosis: potential for prevention of skin carcinogenesis. Nanomedicine. 2015; 11: 1265-1275.

Bangham AD. Lipid bilayers and biomembranes. Annu Rev Biochem. 1972; 41:753-776.

Bangham AD, Standish MM, Weissmann G. The action of steroids and streptolysin $S$ on the permeability of phospholipid structures to cations. J Mol Biol. 1965; 13: 253-259.

Barenholz Y. Doxil(R)--the first FDA-approved nano-drug: lessons learned. J Control Release. 2012; 160: 117-134.

Baselga J, Manikhas A, Cortes J, Llombart A, Roman L, Semiglazov VF, et al. Phase III trial of nonpegylated liposomal doxorubicin in combination with trastuzumab and paclitaxel in HER2-positive metastatic breast cancer. Ann Oncol. 2014; 25: 592-598.

Batist G, Gelmon KA, Chi KN, Miller WH, Jr., Chia SK, Mayer LD, et al. Safety, pharmacokinetics, and efficacy of CPX-1 liposome injection in patients with advanced solid tumors. Clin Cancer Res. 2009; 15: 692-700.

Batist G, Ramakrishnan G, Rao CS, Chandrasekharan A, Gutheil J, Guthrie T, et al. Reduced cardiotoxicity and preserved antitumor efficacy of liposome-encapsulated doxorubicin and cyclophosphamide compared with conventional doxorubicin and cyclophosphamide in a randomized, multicenter trial of metastatic breast cancer. J Clin Oncol. 2001; 19: 14441454.

Berger JL, Smith A, Zorn KK, Sukumvanich P, Olawaiye AB, Kelley J, et al. Outcomes analysis of an alternative formulation of PEGylated liposomal doxorubicin in recurrent epithelial ovarian carcinoma during the drug shortage era. Onco Targets Ther. 2014; 7:1409-1413.

Bhardwaj A, Srivastava SK, Singh S, Arora S, Tyagi $\mathrm{N}$, Andrews J, et al. CXCL12/CXCR4 signaling counteracts docetaxel-induced microtubule stabilization via p21-activated kinase 4dependent activation of LIM domain kinase 1 . Oncotarget. 2014; 5: 11490-11500.

Bitounis D, Fanciullino R, Iliadis A, Ciccolini J. Optimizing Druggability through Liposomal Formulations: New Approaches to an Old Concept. ISRN Pharm. 2012; 2012:738432. doi: 10.5402/2012/738432.

Blade J, Sonneveld P, San Miguel JF, Sutherland HJ, Hajek R, Nagler A, et al. Efficacy and safety of pegylated liposomal Doxorubicin in combination with bortezomib for multiple myeloma: effects of adverse prognostic factors on outcome. Clin Lymphoma Myeloma Leuk. 2011; 11: 44-49.

Bozzuto $G$ and Molinari A. Liposomes as nanomedical devices. Int $J$ Nanomedicine. 2015; 10:975-999.

Bradbury PA and Shepherd FA. Immunotherapy for lung cancer. J Thorac Oncol. 2008; 3: S164S170.

Broglio KR, Stivers DN, Berry DA. Predicting clinical trial results based on announcements of interim analyses. Trials. 2014; 15:73. doi: 10.1186/1745-6215-15-73

Chou H, Lin H, Liu JM. A tale of the two PEGylated liposomal doxorubicins. Onco Targets Ther. 2015; 8:1719-1720.

Cooley T, Henry D, Tonda M, Sun S, O'Connell M, Rackoff W. A randomized, double-blind study of pegylated liposomal doxorubicin for the treatment of AIDS-related Kaposi's sarcoma. Oncologist. 2007; 12: 114-123.

Cortes JE, Goldberg SL, Feldman EJ, Rizzeri DA, Hogge DE, Larson $M$, et al. Phase II, multicenter, randomized trial of CPX-351 (cytarabine:daunorubicin) liposome injection 
versus intensive salvage therapy in adults with first relapse AML. Cancer. 2015; 121: 234-242.

Deshmukh SK, Srivastava SK, Bhardwaj A, Singh AP, Tyagi N, Marimuthu S, et al. Resistin and interleukin-6 exhibit racially-disparate expression in breast cancer patients, display molecular association and promote growth and aggressiveness of tumor cells through STAT3 activation. Oncotarget. 2015; 6: 11231-11241.

Du S and Deng Y. Studies on the encapsulation of oxymatrine into liposomes by ethanol injection and $\mathrm{pH}$ gradient method. Drug Dev Ind Pharm. 2006; 32: 791-797.

Ellens H, Bentz J, Szoka FC. Destabilization of phosphatidylethanolamine liposomes at the hexagonal phase transition temperature. Biochemistry. 1986; 25: 285-294.

Fantini M, Gianni L, Santelmo C, Drudi F, Castellani C, Affatato A, et al. Lipoplatin treatment in lung and breast cancer. Chemother Res Pract. 2011; 2011:125192. doi: 10.1155/2011/125192.

Farge E and Devaux PF. Shape changes of giant liposomes induced by an asymmetric transmembrane distribution of phospholipids. Biophys J. 1992; 61: 347-357.

Fitzmaurice C, Dicker D, Pain A, Hamavid H, Moradi-Lakeh M, MacIntyre MF, et al. The Global Burden of Cancer 2013. JAMA Oncol. 2015; 1: 505-527.

Geretti E, Leonard SC, Dumont N, Lee H, Zheng J, De SR, et al. Cyclophosphamide-mediated tumor priming for enhanced delivery and antitumor activity of HER2-targeted liposomal doxorubicin (MM-302). Mol Cancer Ther. 2015.

Goldberg MS, Hook SS, Wang AZ, Bulte JW, Patri AK, Uckun FM, et al. Biotargeted nanomedicines for cancer: six tenets before you begin. Nanomedicine (Lond). 2013; 8: 299-308.

Gregoriadis G and Florence AT. Liposomes in drug delivery. Clinical, diagnostic and ophthalmic potential. Drugs. 1993; 45: 15-28.

Haran G, Cohen R, Bar LK, Barenholz Y. Transmembrane ammonium sulfate gradients in liposomes produce efficient and stable entrapment of amphipathic weak bases. Biochim Biophys Acta. 1993;1151: 201-215.

Immordino ML, Dosio F, Cattel L. Stealth liposomes: review of the basic science, rationale, and clinical applications, existing and potential. Int J Nanomedicine. 2006; 1: 297-315.

Iwamoto T. Clinical application of drug delivery systems in cancer chemotherapy: review of the efficacy and side effects of approved drugs. Biol Pharm Bull. 2013; 36: 715-718.

James ND, Coker RJ, Tomlinson D, Harris JR, Gompels M, Pinching AJ, et al. Liposomal doxorubicin (Doxil): an effective new treatment for Kaposi's sarcoma in AIDS. Clin Oncol ( $R$ Coll Radiol ). 1994; 6: 294-296.

Jing Li, Xuling Wang, Ting Zhang, Chunling Wang, Zhenjun Huang, Xiang Luo, et al. A review on phospholipids and their main applications in drug delivery systems. Asian Journal of Pharmaceutical Sciences. 2015; 10: 81-98.

Kirby C and Gregoriadis G. The effect of the cholesterol content of small unilamellar liposomes on the fate of their lipid components in vitro. Life Sci. 1980; 27: 2223-2230.

Ko AH, Tempero MA, Shan YS, Su WC, Lin YL, Dito E, et al. A multinational phase 2 study of nanoliposomal irinotecan sucrosofate (PEP02, MM-398) for patients with gemcitabinerefractory metastatic pancreatic cancer. $\mathrm{Br} J$ Cancer. 2013;109: 920-925.

Lancet JE, Cortes JE, Hogge DE, Tallman MS, Kovacsovics TJ, Damon LE, et al. Phase 2 trial of CPX-351, a fixed 5:1 molar ratio of cytarabine/daunorubicin, vs cytarabine/daunorubicin in older adults with untreated AML. Blood. 2014; 123: 3239-3246.

Laouini A, Jaafar-Maalej C, Limayem-Blouza I, Sfar S, Charcosset C, Fessi H. Preparation, Characterization and Application of Liposomes: State of the Art. Journal of Colloidal Science and Biotechnology. 2012; 1: 147-168.

Lasic DD, Frederik PM, Stuart MC, Barenholz Y, McIntosh TJ. Gelation of liposome interior. A novel method for drug encapsulation. FEBS Lett. 1992; 312: 255-258.

MacDonald V. Chemotherapy: managing side effects and safe handling. Can Vet J. 2009; 50: 665668.

Magarkar A, Dhawan V, Kallinteri P, Viitala T, Elmowafy M, Rog T, et al. Cholesterol level affects surface charge of lipid membranes in saline solution. Sci Rep. 2014; 4: 5005. doi: 10.1038/srep05005.

Manes S and Martinez A. Cholesterol domains regulate the actin cytoskeleton at the leading edge of moving cells. Trends Cell Biol. 2004; 14: 275-278.

Marques-Gallego $\mathrm{P}$ and de Kroon AI. Ligation strategies for targeting liposomal nanocarriers. Biomed Res Int. 2014; 2014:129458. doi: $10.1155 / 2014 / 129458$.

Miller CR, Bondurant B, McLean SD, McGovern KA, O'Brien DF. Liposome-cell interactions in vitro: effect of liposome surface charge on the binding and endocytosis of conventional and sterically stabilized liposomes. Biochemistry. 1998; 37: 12875-12883.

Muggia FM. Clinical efficacy and prospects for use of pegylated liposomal doxorubicin in the treatment of ovarian and breast cancers. Drugs. 1997; 54 Suppl 4:22-29. 
Pattni BS, Chupin VV, Torchilin VP. New Developments in Liposomal Drug Delivery. Chem Rev. 2015.

Petre CE and Dittmer DP. Liposomal daunorubicin as treatment for Kaposi's sarcoma. Int $J$ Nanomedicine. 2007; 2: 277-288.

Poon RT and Borys N. Lyso-thermosensitive liposomal doxorubicin: an adjuvant to increase the cure rate of radiofrequency ablation in liver cancer. Future Oncol. 2011; 7: 937-945.

Qiu L, Jing N, Jin Y. Preparation and in vitro evaluation of liposomal chloroquine diphosphate loaded by a transmembrane $\mathrm{pH}$-gradient method. Int J Pharm. 2008; 361: 56-63.

Ramirez LY, Huestis SE, Yap TY, Zyzanski S, Drotar D, Kodish E. Potential chemotherapy side effects: what do oncologists tell parents? Pediatr Blood Cancer. 2009; 52: 497-502.

Riviere K, Kieler-Ferguson HM, Jerger K, Szoka FC, Jr. Anti-tumor activity of liposome encapsulated fluoroorotic acid as a single agent and in combination with liposome irinotecan. J Control Release. 2011; 153: 288-296.

Rodriguez MA, Pytlik R, Kozak T, Chhanabhai M, Gascoyne R, Lu B, et al. Vincristine sulfate liposomes injection (Marqibo) in heavily pretreated patients with refractory aggressive non-Hodgkin lymphoma: report of the pivotal phase 2 study. Cancer. 2009; 115: 3475-3482.

Roy AC, Park SR, Cunningham D, Kang YK, Chao $\mathrm{Y}$, Chen LT, et al. A randomized phase II study of PEP02 (MM-398), irinotecan or docetaxel as a second-line therapy in patients with locally advanced or metastatic gastric or gastrooesophageal junction adenocarcinoma. Ann Oncol. 2013; 24: 1567-1573.

Saif MW. MM-398 achieves primary endpoint of overall survival in phase III study in patients with gemcitabine refractory metastatic pancreatic cancer. JOP. 2014; 15: 278-279.

Sarris AH, Hagemeister F, Romaguera J, Rodriguez MA, McLaughlin P, Tsimberidou AM, et al. Liposomal vincristine in relapsed non-Hodgkin's lymphomas: early results of an ongoing phase II trial. Ann Oncol. 2000; 11: 69-72.

Sessa G and Weissmann G. Phospholipid spherules (liposomes) as a model for biological membranes. J Lipid Res. 1968; 9: 310-318.

Simon SA, McIntosh TJ, Latorre R. Influence of cholesterol on water penetration into bilayers. Science. 1982; 216: 65-67.

Srivastava SK, Bhardwaj A, Arora S, Tyagi N, Singh $\mathrm{AP}$, Carter JE, et al. Interleukin-8 is a key mediator of FKBP51-induced melanoma growth, angiogenesis and metastasis. $\mathrm{Br} J$ Cancer. 2015a; 112: 1772-1781.

Srivastava SK, Bhardwaj A, Arora S, Tyagi N, Singh $\mathrm{S}$, Andrews J, et al. MicroRNA-345 induces apoptosis in pancreatic cancer cells through potentiation of caspase-dependent and independent pathways. $\mathrm{Br} J$ Cancer. 2015b; 113; 660-668.

Staruch R, Chopra R, Hynynen K. Localised drug release using MRI-controlled focused ultrasound hyperthermia. Int J Hyperthermia. 2011; 27: 156-171.

Stewart, B. W., Wild, C. P. e. World Cancer Report 2014. Lyon, France: International Agency for Research on Cancer. 2014. http://www.iarc.fr/en/publications/books/wcr/wc r-order.php.

Sutradhar KB and Lutful A. Nanotechnology in Cancer Drug Delivery and Selective Targeting. ISRN Nanotech. 2014; 2014: 939378. doi: 10.1155/2014/939378.

Suzuki R, Takizawa T, Kuwata Y, Mutoh M, Ishiguro $\mathrm{N}$, Utoguchi $\mathrm{N}$, et al. Effective antitumor activity of oxaliplatin encapsulated in transferrin-PEG-liposome. Int J Pharm. 2008; 346: 143-150.

Torre LA, Bray F, Siegel RL, Ferlay J, LortetTieulent J, Jemal A. Global cancer statistics, 2012. CA Cancer J Clin. 2015; 65: 87-108.

Tyagi N, Bhardwaj A, Singh AP, McClellan S, Carter JE, Singh S. p-21 activated kinase 4 promotes proliferation and survival of pancreatic cancer cells through AKT- and ERK-dependent activation of NF-kappaB pathway. Oncotarget. 2014; 5: 8778-8789.

Tyagi N and Ghosh PC. Folate receptor mediated targeted delivery of ricin entrapped into sterically stabilized liposomes to human epidermoid carcinoma (KB) cells: effect of monensin intercalated into folate-tagged liposomes. Eur J Pharm Sci. 2011; 43: 343-353.

Tyagi N, Rathore SS, Ghosh PC. Enhanced killing of human epidermoid carcinoma (KB) cells by treatment with ricin encapsulated into sterically stabilized liposomes in combination with monensin. Drug Deliv. 2011; 18: 394-404.

Tyagi N, Rathore SS, Ghosh PC. Efficacy of Liposomal Monensin on the Enhancement of the Antitumour Activity of Liposomal Ricin in Human Epidermoid Carcinoma (KB) Cells. Indian J Pharm Sci. 2013; 75: 16-22.

Received: 24-Aug - 2015 Accepted: 08-Oct-2015 